\title{
Sur les traces de l'emprunt caché
}

Approche phonographique, morphologique et sémantique d'un gallicisme en espagnol

\section{Stéphane Oury}

\section{(2) OpenEdition}

1 Journals

Édition électronique

URL : https://journals.openedition.org/cher/11353

DOI : 10.4000/cher.11353

ISSN : 2803-5992

\section{Éditeur}

Presses universitaires de Strasbourg

\section{Édition imprimée}

Date de publication : 1 décembre 2012

Pagination : 23-37

ISBN : 978-2-35410-049-0

ISSN : 1968-035X

\section{Référence électronique}

Stéphane Oury, "Sur les traces de l'emprunt caché », reCHERches [En ligne], 9 | 2012, mis en ligne le 14 février 2022, consulté le 16 février 2022. URL : http://journals.openedition.org/cher/11353 ; DOI : https://doi.org/10.4000/cher.11353

\section{cc) (†) (ㅇ)}

Ce(tte) œuvre est mise à disposition selon les termes de la Licence Creative Commons Attribution Pas d'Utilisation Commerciale - Partage dans les Mêmes Conditions 4.0 International. 


\title{
Sur les traces de l'emprunt caché. Approche phonographique, morphologique et sémantique d'un gallicisme en espagnol
}

\author{
StÉPHANE OURY \\ Université Paul Verlaine - Metz
}

$\mathrm{D}$ ans le cadre de la journée d'études "Empreintes, emprunts», nous avons choisi d'axer notre travail sur une forme de néologisme très féconde: l'emprunt et, plus précisément, un gallicisme supposé: caché. Il faut donc prendre caché dans sa valeur autonymique et lire le titre de notre communication de la façon suivante: "Sur les traces de l'emprunt [kaĉé], approche phonographique, morphologique et sémantique d'un gallicisme en espagnol».

Le gallicisme (avec plus de 2000 vocables $^{1}$ ), après l'arabisme (environ 4000 vocables selon Ramón Menéndez Pidal et Rafael Cano Aguilar ${ }^{2}$ ) et avant toute autre langue, a marqué l'espagnol de son empreinte, ce qui nous ramène naturellement à l'objet de cette journée.

Nous sommes partis du postulat suivant:

Postulat: caché semble fréquent dans la langue espagnole actuelle et présent dans toutes les aires hispaniques. Sa graphie et prosodie laissent croire qu'il s'agit d'un gallicisme.

1 Une étude menée à l'occasion du colloque de Nancy le 28 mai 2010: Emprunts et transferts culturels et leur représentation dans le monde luso hispanophone, portant sur un large échantillon de la lexicographie espagnole et latino-américaine, révèle 2404 vocables hors dérivés (1758 DRAE + 348 lexicographie espagnole descriptive + 298 lexicographie latino-américaine).

2 Respectivement in Gramática histórica del español (1999: 24-25) et El español a través de los siglos (1988: 53). 
Nous allons essayer de le vérifier en mettant en œuvre une méthodologie avant d'observer les résultats obtenus et de nous interroger sur l'adaptation linguistique dudit vocable dans sa langue d'accueil.

\section{Méthodologie}

Identifier la ou les formes concernées, interroger l'étymologie du vocable, le repérer dans la langue et le discours, cerner son degré d'intégration, le localiser géographiquement sont autant d'impératifs qui vont exiger le recours à des outils divers.

\subsection{Un lexème ou plusieurs?}

Notre réponse dépendra à la fois du traitement lexicographique réservé à la/les forme(s) rencontrée(s) et au rapport sémantique existant entre elles.

\section{2. Étymologie}

Nous aurons recours au dictionnaire étymologique de référence du gallo-roman: le FEW (Französisches Etymologisches Wörterbuch) pour l'étymologie première et aux dictionnaires unilingues espagnols faisant autorité et offrant une rubrique étymologique pour établir le canal d'entrée en espagnol (notons que le vocable, assez récent en espagnol, n'est pas recensé par Corominas ni Bénaben).

\subsection{Repérage du lexème dans la langue actuelle et degré d'intégration}

Afin de vérifier la présence du vocable dans la langue ainsi que son degré d'intégration (barbarisme, xénisme, emprunt assimilé et normatif), nous avons choisi un corpus de dictionnaires représentatifs de la langue espagnole, à la fois descriptifs et normatifs.

Nous avons en effet interrogé un panel de dictionnaires espagnols se voulant représentatif de la langue actuelle. Nous avons essayé de réduire par un spectre large au maximum les facteurs de distorsion habituels (manque de réactivité, hyperspécialisation, hypermodernité ou hyperconservatisme³). Les dictionnaires retenus ont été les suivants:

3 Certains, comme Unamuno pensent que le dictionnaire est le «cimetière des mots » (¡Qué cementerio el diccionario!)», idée reprise par N. Clarasó: «Diccionario. Tumba donde yacen los cadáveres de las palabras que ya nadie usa; y donde no queda sitio para las palabras nuevas que usa todo el mundo" in Diccionario humorístico (1997: 93). En effet, 
- le Diccionario de la lengua española de la Real Academia Española, (22e éd, Madrid, Espasa Calpe, 2001, 2 volumes) que nous abrègerons désormais en DRAE;

- le Diccionario de Uso del Español de María Moliner, (2e edition revue et augmentée, Madrid, Gredos, 2007, 2 volumes) que nous abrègerons en DUE;

- le Diccionario del Español Actual de O. Andrés, G. Ramos et M. Seco (Madrid, Aguilar, 1999, 2 volumes) que nous abrègerons en DEA;

- le Diccionario de uso del español actual Clave, (Madrid, S. M., 1996) que nous abrègerons en Clave;

- le Diccionario Salamanca de la lengua española, (Salamanca, Santillana, 2006) que nous abrègerons en SAL;

- le Diccionario Panhispánico de Dudas de la Real Academia española (Madrid, Santillana, 2005) que nous abrègerons en DPD.

\subsection{Repérage du lexème dans le discours et localisation dans l'espace}

Pour observer le vocable évoluer en discours et pour essayer de cerner sa distribution diatopique, nous avons eu recours à un formidable outil proposé par l'Académie Royale de Langue Espagnole, à savoir le CREA, sigle pour Corpus de Referencia del Español Actual.

En effet, il y a quelques années, la RAE s'est dotée du CREA. Le volume des occurrences (160 millions), la modernité (1975-2004), l'actualisation permanente (la dernière datant de juin 2008 avec 3,5 millions d'ajouts, pour beaucoup issus de la presse latino-américaine), la variété des supports ( $90 \%$ issus de l'écrit et $10 \%$ de l'oral, $50 \%$ d'occurrences provenant de l'Espagne et $50 \%$ de l'Amérique, livres, journaux, radio, télévision, grande diversité thématique) offre de précieuses indications au linguiste (fréquence, localisation, emploi en contexte).

Notons que la RAE annonce un Corpus del Español del XXI qui prendra davantage en compte l'espagnol d'Amérique par rapport à l'espagnol péninsulaire $(70 \% / 30 \%$ contre $50 \% / 50 \%$ actuellement), source rae.es > Real Academia Española $>$ Banco de datos $>$ El Corpus del Siglo XxI.

Le CREA nous servira donc à établir un relevé des occurrences en discours. Une lecture fine permettra d'isoler les différentes acceptions et d'en localiser les aires d'emploi.

l'écart entre l'apparition d'un vocable dans l'usage et son apparition dans la lexicographie peut être important. 


\section{Résultats obtenus et exploitation des données}

\subsection{Trace dans la lexicographie actuelle}

La présence du vocable est attestée dans les dictionnaires DRAE, DPD, Clave, DEA, DUE, SAL, c'est-à-dire dans la lexicographie descriptive, mais aussi prescriptive et normative (DRAE, DPD). Le vocable jouit sans doute d'une certaine vitalité et a dépassé le stade de barbarisme* (emploi abusif d'un point de vue normatif) et de xénisme (citation ou forme non assimilée) pour devenir un gallicisme assimilé.

\subsection{Occurrences CREA et localisation}

Le relevé total cumulé est de 225 occurrences répondant à la distribution suivante:

- 180 occurrences de la forme caché

- 32 de la forme cachet

- 13 de la forme cache.

Notons que ce relevé a été épuré de tout homonyme parasite (caché $<$ prétérit, première personne du singulier du verbe cachar, cache < catch, par exemple).

Ce nombre d'occurrences situe le vocable parmi les $20 \%$ de gallicismes les plus usités en espagnol. Voici, pour indication des exemples de gallicismes de fréquence voisine: mousse (75), voyeur (88), afiche (122), caché (180), impasse (232), affaire (243), dossier (288).

La localisation des occurrences est la suivante:

- 70/30 dans le rapport péninsule/ Amérique latine (où toutes les aires sont représentées, du Río de la Plata à l'Amérique centrale en passant par les Andes) pour la forme caché.

- 50/50 pour la forme cachet avec une diffusion assez homogène et un pic en Argentine.

- $100 \%$ des occurrences de cache en Amérique latine.

Le rapport global est d'environ 60/40 à l'avantage de l'Espagne. Eu égard au corpus du CREA, nous pouvons donc parler d'une diffusion généralisée et assez homogène du vocable.

4 Il était encore absent dans le DRAE 21 (1992). 


\subsection{Homonymie ou polysémie?}

Les trois formes rencontrées nous invitent à nous interroger sur leur identification: s'agit-il de formes homonymes ou d'un seul lexème polysémique: 1, 2 ou 3 lexèmes, en somme?

On relève trois sens récurrents que l'on peut classer de la façon suivante:

caché $1 . \quad$ "rétribution d'un artiste"

caché 1 bis. "distinction, charme"

caché $2 . \quad$ "mémoire cache, antémémoire"

Les acceptions 1. et 1 bis. («rétribution d'un artiste» et «distinction») dérivant d'une même acception première (outil puis trace de celui-ci, cf. supra, Etymologie), nous faisons le choix de considérer 1. et 1bis. comme les acceptions d'un même lexème polysémique (notons que le DEA considère pour sa part qu'il s'agit de 2 lexèmes différents). 2. représentera pour nous un lexème (adjectif ou substantif) distinct.

Le canal d'entrée des vocables n'est au reste pas le même: caché 1 . et 1 bis. sont entrès en espagnol directement par le français alors que caché 2 . y est entré par l'anglo-américain qui l'a précédemment emprunté au français.

\section{Adaptation linguistique}

Voyons comment ces vocables exogènes ont réussi à se couler dans les moules phonologique, graphique, morphologique et sémantique de leur langue d'adoption.

\subsection{Adaptation phonographique}

Il apparaît ici une forte tendance (presque une constante) parmi les emprunts: la cohabitation de graphies multiples pouvant traduire différents stades d'assimilation, des canaux de pénétration différents et parfois concomitants ou des sphères d'emploi aux motivations différenciées.

\subsubsection{Priorité graphique ou phonétique}

Cachet est un emprunt brut, oxyton témoignant d'une fidélité prosodique et graphique. Le canal d'entrée a sans doute été l'écrit. Cette forme se pare par ailleurs d'un certain prestige, un vernis culturel, un signum de classe diraient certains linguistes qui peuvent être l'une des motivations de l'emprunt. 
Notons qu'en Argentine, la prononciation majoritaire de cachet est [kašé] conjuguant par le fait, fidélité graphique et phonétique et affichant par là même sa volonté de se démarquer de la langue de l'ancien colonisateur en s'affranchissant de son filtre phonétique.

Caché est un emprunt adapté, un singulier régressif issu d'une simplification d'un pluriel problématique (cachets $>$ cachés) plus conforme aux schémas morphologiques de l'espagnol, comme cela a été le cas pour d'autres gallicismes à la finale en "-et» (chalé, carné...). La fidélité prosodique (mot oxyton) et phonétique (son [e] conservé) laissent à penser que le canal d'entrée a été oral. Il s'inscrit parfaitement dans la tradition d'assimilation de l'espagnol (cf. fútbol, cóctel).

\subsubsection{La recherche du modèle idéal}

Cache est la forme la plus adaptée (mais sans doute la moins valorisée socialement) qui tend vers le schéma phonétique vers lequel tend idéalement tout vocable en espagnol.

Bertil Malmberg 5 souligne, à propos de l'espagnol, l'importance du modèle syllabique castillan de type CV/CV (consonne-voyelle/ consonne-voyelle). Ce modèle représente, selon lui, la structure idéale vers laquelle tendent inconsciemment les locuteurs. A. Quilis ajoute en ce qui le concerne: «En español, las sílabas abiertas son las más numerosas, representan el $66,19 \% »^{6}$. Enfin, R. Omnès ${ }^{7}$ insiste sur la prééminence du schéma prosodique paroxyton ( $74 \%$ des mots dans Cinco horas con Mario de Miguel Delibes).

Le vocable espagnol idéal ressemblerait donc à un dissyllabe paroxyton de structure syllabique ouverte de type $\mathrm{CV} / \mathrm{CV}$. Il est intéressant de voir comment l'espagnol s'efforce donc de couler l'emprunt (mais pas seulement ${ }^{8}$ ) dans son moule accentuel et c'est le cas ici de cache. Mais il est d'autres pressions (pression sociale, poids de l'écrit, quête de démarcation, d'exotisme phonographique) qui éloignent, à dessein bien souvent, l'emprunt

5 Malmberg B., Phonétique générale et romane (1971: 470).

6 Quilis A., Tratado de fonética y fonología (1993: 67). Il est à noter qu'il ne précise pas son corpus. Dans le doute, on préférera les statistiques de R. Omnès qui ne comptabilisent que les mots à lexèmes et dont le corpus est un texte se rapprochant de la langue de conversation.

7 Omnès R., Phonétique, phonologie, orthographe et prononciation de l'espagnol (1995: 26).

8 Pensons aux formes courtes créées par la langue familière, par apocope ou aphérèse, (profe, boli, bici, chacha, tano) et qui respectent ces normes. 
de ce schéma. R. Omnès ajoute au sujet des formes «exotiques» qu'elles sont davantage possibles chez un locuteur cultivé:

«Statistiquement les syllabes ouvertes dominent largement et les mots de structure CVCV sont extrêmement nombreux. D'autres structures sont acceptables et donc "tolérées" [...]. Certains mots éloignés du modèle pourront s'imposer notamment chez les locuteurs cultivés, disposant d'un arsenal phonique plus riche que d'autres gens. Ce sont les analphabètes qui obéissent le mieux au modèle syllabique » ${ }^{9}$

\subsubsection{Forme dominante}

Le recensement lexicographique opéré révèle la présence de caché dans les dictionnaires DRAE, DUE, DEA, DPD, SAL et celle de cachet dans les dictionnaires Clave, DUE, DEA et SAL.

La forme cache ne se retrouve dans aucun dictionnaire.

La forme dominante s'avère être caché (dans un ratio de 55/45 par rapport à cachet).

L'inventaire réalisé grâce au CREA fait apparaître les résultats suivants: nombre d'occurrences: caché: 180 / cachet: 32 / cache: 13 . Ces résultats sont à étudier par acception (voir tableau ci-après):

\begin{tabular}{|l|c|c|c|c|}
\hline Forme/acception & 1. «rétribution» & 1bis. «distinction» & 2. «antémémoire» & Total \\
\hline Caché & 35 & 49 & 96 & 180 \\
\hline Cachet & 30 & 2 & 0 & 32 \\
\hline Cache & 1 & 1 & 11 & 13 \\
\hline \multicolumn{1}{|c|}{ Total } & 66 & 52 & 107 & 225 \\
\hline
\end{tabular}

Il est à noter que les trois formes ne se limitent pas pour chacune d'entre elles à une acception. Ce phénomène de variante graphique ou allomorphe est fréquent en matière d'emprunt. Il ressort de ce relevé que la forme dominante est caché. Elle est à même de dire les trois acceptions, mais dit majoritairement "mémoire cache» et «distinction». La forme cachet ne signifie jamais "mémoire cache» et se révèle de fréquence équivalente à caché dans le sens de "rétribution d'un artiste». La forme cache, très minoritaire prend majoritairement le sens de «mémoire cache».

9 Omnès 1995: 27. 


\subsection{Adaptation morphologique}

\subsubsection{Les formes (relativement) académiques}

El caché ou ses variantes allomorphes cachet/cache se coulent assez bien dans les moules de la langue d'accueil (hormis le problème du pluriel de cachet mentionné précédemment).

\subsubsection{Une évolution singulière}

La forme rencontrée la caché, au féminin ne laisse de nous interroger. Il y a eu changement de catégorie grammaticale en suivant le processus suivant: adjectif variable cachée $>$ adjectif invariable caché $>$ substantif avec maintien majoritaire du genre féminin, par ellipse du substantif «(mémoire) cachée» avec perte du morphème de genre « $\mathrm{e}$ ».

Or si l'origine française du principe même d' «antémémoire» (apparu dans les années 1960 à Grenoble) et de la lexie complexe afférente «mémoire cachée » sont attestées, les différentes langues semblent l'accueillir aujourd'hui depuis l'anglais.

Le français lui-même dans un phénomène connu sous le nom d'allerretour (cf. «tennis » < l'ancien français «tenetz », « toast » < de l'ancien français «toster», ou «alligator» < d'une déformation anglo-américaine de l'espagnol «el lagarto») semble aujourd'hui préférer la forme «mémoire cache».

Ces éléments nous conduisent à l'interprétation suivante: peut-être que caché n'est pas (n'est plus) un adjectif invariable mais plutôt un substantif en apposition (comprendre "cache» et non "caché» comme en anglais cache memory ou en français «mémoire cache» ou «antémémoire») qui peut fonctionner en complément du substantif si celui-ci est présent: memoria caché, archivo caché ou en l'absence de celui-ci (ellipse): la caché, el caché. La fluctuation de genre peut alors s'expliquer par le genre du substantif sousentendu (majoritairement la memoria, mais aussi el sistema, el archivo). Notons que l'omission du substantif memory a cours également en anglais cache pour cache memory ou en français «le cache» suivant un principe bien connu: celui de l'économie linguistique.

\subsection{Adaptation sémantique}

\subsection{1. Étymologie}

Cachet est dérivé du radical du verbe "cacher»+ suffixe «-et» (suffixe traduisant l'objet avec lequel l'action s'accomplit) selon le Französisches 
Etymologisches Wörterbuch (FEW) du suisse Walther Von Wartburg (t.2, 1, p. 807).

La forme procède du latin *coacticare "comprimer, serrer", forme renforcée du latin coactare "contraindre" fréquentatif de cogere de même sens. De coactare, ont dérivé les formes méridionales de type cacha "écraser, broyer, presser, blesser». Ce même sens de "écraser» est attesté dès le $\mathrm{XII}^{\mathrm{e}}$ siècle en ancien français escachier. Le sens de "dissimuler", peu fréquent jusqu'au XVI ${ }^{e}$ siècle, est dérivé de celui de "presser, comprimer».

\subsection{2. Évolution en français}

Le FEW et le TLF (Trésor de la langue française, 1971-1994, Paris, Gallimard, 16 volumes) s.v., s'accordent à signaler l'évolution suivante: l'acception première est "petit sceau » $\left(\mathrm{XV}^{\mathrm{e}}\right)$, «outil» puis dès le $\mathrm{XVI}^{\mathrm{e}}$ siècle, une deuxième acception: "trace de celui-ci» dans un rapport de cause à effet puis une troisième: "rétribution", au XVII ${ }^{\mathrm{e}}$ siècle (initialement justifié par un cachet sur une carte) puis une quatrième, par abstraction et généralisation: "toute trace singulière" au XIX ${ }^{e}$ siècle, «relief, singularité, distinction" et enfin une cinquième et dernière: "capsule» sens dérivé du relief (comprimé) à la fin du XIX ${ }^{e}$ siècle.

\subsubsection{Emprunt partiel au français}

Les rubriques étymologiques des dictionnaires consultés (en plus des ressemblances formelles et des dates de première occurrence dans chacune des langues) nous confirment bien l'origine française du vocable: «caché. 1. Adaptación gráfica de la voz francesa cachet» (DPD, s.v.), "caché. Del fr. cachet» (DRAE 22, s.v.). Il s'agit donc bien d'un gallicisme.

A la lumière de ces définitions, nous voyons que l'emprunt en espagnol n'est que partiel: il se limite aux acceptions 3. et 4. devenues 1 . et 1bis. dans notre exposé (quelques rares occurrences de 2. qui restent des xénismes, à savoir un emprunt non assimilé).

Une approche diachronique nous permet une datation des premières occurrences ainsi qu'un aperçu de la chronologie des acceptions. Les premières occurrences remontent à la fin $\mathrm{du} \mathrm{XIX}^{\mathrm{e}}$ siècle et l'acception dominante est "distinction" (source CORDE, acronyme pour CORpus Diacrónico del Español, banque de données linguistique de la RAE). 


\subsubsection{Emprunt direct et indirect: gallicisme et/ou anglo-américanisme?}

Si la filiation de caché 1 . et 1 bis. est entendue, le cas est moins aisé pour caché 2 .

Caché 2. < anglais "cache memory" < français «mémoire cachée» ou par calque: cache memory à partir de lexèmes préexistant en anglais mais dont le premier élément «cache» a été emprunté au français "cache» déverbal du verbe "cacher» (attesté au féminin dès le $\mathrm{XVI}^{\mathrm{e}}$ siècle et au masculin dès le XIXe siècle. Plus récemment, il apparaît comme élément de composition, toutes formes attestées en anglais: cache $>$ un cache, une cache, cache-sexe). Rappelons que l'anglais offre sans doute le plus gros réservoir de gallicismes et que, outre les formes précitées, il emploie également cachet dans le sens de «distinction».

Quoi qu'il en soit, caché 2. procède, au moins indirectement, du verbe français «cacher» (dans son sens tardif d' «occulter» qui s'étend à partir $\mathrm{du} \mathrm{XVI}{ }^{\mathrm{e}}$ siècle et supplante "escondre, musser» de l'ancien français (TLF et FEW s.v.).

"Mémoire cachée» a sans doute été calqué par l'anglais et diffusé par celui-ci. L'espagnol a emprunté la lexie à l'anglais en lui imposant, par analogie homonymique (caché < cachet), un schéma accentuel oxyton.

C'est donc un cas d'anglicisme ou anglo-américanisme immédiat en synchronie (cf. DPD) ou gallicisme indirect en diachronie. Il s'agit d'un anglicisme lexical mais également d'un gallicisme prosodique. Nous pourrions donc ici parler de l'emprunt [kašé] tout autant que de l'emprunt [kaćé] (voir titre et introduction).

Notons enfin ici que l'anglo-américain est un formidable vecteur ou réactivateur de gallicismes. Il fait entrer des formes nouvelles ou revitalise des formes préexistantes (restaurant, croissant..., formes qui sont souvent devenues des internationalismes). Cachet (distinction) attesté en ce sens en anglais, a pu bénéficier de cet effet de surexposition eu égard au rayonnement de cette langue.

\subsubsection{Acception dominante}

Dans la lexicographie:

- $100 \%$ pour les acceptions 1. «rétribution d'un artiste» et 1 bis. «distinction» présentes dans tous les dictionnaires (6/6). 
- 33\% pour l'acception 2. "antémémoire» présent dans DPD et DEA mais absent de DRAE 22, de Clave, de DUE et de SAL (2/6).

D'après les occurrences CREA, c'est inversement proportionnel: «Antémémoire» ou caché 2. est dominant (107 occurrences) devant «rétribution» ou caché 1 (66) et «distinction» ou caché 1 bis. (52).

La représentation massive dans l'usage et sous-représentation dans la lexicographie de caché 2 . peuvent s'expliquer dans la mesure où il s'agit d'un emprunt récent, comblant une lacune lexicale (nouvelle réalité et besoin dénominatif à satisfaire) et de surcroît dans un domaine en pleine expansion (l'informatique) et véhiculé par l'anglais.

\section{Conclusion}

Au début de notre exposé, nous avons joué sur les mots. En fait, il n'y avait pas loin de l'emprunt caché [kacée à l'emprunt caché [kašé]. Et la radiographie de cet emprunt nous montre que l'affaire est plus complexe qu'il n'y parait: la forme cache en réalité trois acceptions et deux catégories grammaticales différentes.

Les motivations sont diverses mais réelles: l'économie linguistique (caché est plus efficace d'un point de vue graphique et phonique que "distinción" ou "cotización») conjuguée au prestige de la forme française, a motivé les deux premières formes. Le besoin linguistique (combler une lacune lexicale) et le poids de l'anglais dans le domaine informatique ont fait émerger la troisième. Ces raisons devraient suffire à assurer leur pérennité (ils ont d'ailleurs dépassé le stade de xénismes pour devenir des emprunts assimilés et leur emploi n'est pas/ plus fustigé par les ouvrages normatifs consultés).

Leur itinéraire linguistique est varié (emprunt partiel, direct et indirect, brut et adapté, relayé par l'anglais) et se révèle être assez représentatif du phénomène de transfert linguistique dans sa pluralité.

\section{Bibliographie}

Andrés O., Ramos G., Seco M., 1999, Diccionario del Español Actual, Madrid, Aguilar, 2 volumes.

Cano Aguilar R., 1998, El español a través de los siglos, Madrid, Arco Libros.

Diccionario de la lengua española de la Real Academia Española, 2001, Madrid, Espasa Calpe, 2 volumes. 
Diccionario de uso del español actual Clave, 1996, Madrid, S. M.

Diccionario Panhispánico de Dudas de la Real Academia española 2005, Madrid, Santillana.

Diccionario Salamanca de la lengua española, 2006, Salamanca, Santillana.

Malmberg B., 1971, Phonétique générale et romane, Paris, Mouton.

Menéndez Pidal R., 1999, Gramática histórica del español, Madrid, Espasa Calpe.

Moliner M., 2007, Diccionario de Uso del Español, 2e edition revue et augmentée, Madrid, Gredos, 2 volumes.

Omnès R., 1995, Phonétique, phonologie, orthographe et prononciation de l'espagnol, Paris, Nathan.

Quilis A., 1993, Tratado de fonética y fonología, Madrid, Gredos.

Trésor de la langue française, 1971-1994, Paris, Gallimard, 16 volumes.

Von Wartburg W., 1948, Französisches Etymologisches Wörterbuch, Tübingen, J.C.B. Mohr.

\section{Annexes}

Doc. 1 : entrée caché in DRAE 22a ed. (2001)

Artículo enmendado. Avance de la vigésima tercera edición

caché. (Del fr. cachet).

1. m. Distinción, elegancia. 2. m. Cotización de un artista del espectáculo o de ciertos profesionales que actúan en público. 3. f. Inform. Memoria de acceso rápido de un computador, que guarda temporalmente las últimas informaciones procesadas. U. t. c. adj. Memoria caché. caerse el $\sim$. 1. loc. verb. Cuba. Perder el prestigio o la fama.

darse $\sim$ alguien. 1. loc. verb. coloq. Cuba, El Salv. y Hond. Darse importancia, bien por el modo de vestir, bien por gestos o palabras.

Doc. 2 : entrée caché in DPD (2005)

caché.

1. Adaptación gráfica de la voz francesa cachet, usada en español con los sentidos de 'distinción o elegancia': "Hay quien nace [...] con estilo y caché» (Vergés Cenizas [R. Dom. 1980]); y 'cotización o remuneración de un artista': "¿Qué méritos reúne el retoño de la cantante [...] para tener un caché en televisión de 1500000 pesetas?» (Díaz Televisión [Esp. 1999]). En ambos casos es masculino, por lo que no debe confundirse con el uso, como sustantivo femenino, del adjetivo caché $(\rightarrow 2)$. Su plural es cachés ( $\rightarrow$ PLURAL, $1 \mathrm{a}$ ): «Los cachés de los artistas han subido de un modo espectacular» (Abc [Esp.] 6.12.96). Aunque se admite el uso del galicismo adaptado, no hay que olvidar que existen términos españoles equivalentes, como elegancia o distinción, para el primer sentido, y cotización, para el segundo.

2. Se usa en informática, como adjetivo invariable, para referirse a la memoria de rápido acceso, situada entre el procesador y la memoria principal: "Existen dos tipos de memoria caché: primaria y secundaria» (Pimentel Multimedia [Perú 1997]). También se emplea como sustantivo femenino: "Un genuino Pentium Pro, a $200 \mathrm{MHz}$ de velocidad, con [...] caché interna» (Mundo [Esp.] 13.4.97). En este caso, es voz tomada del inglés cache (memory), 
con acentuación aguda por influjo del galicismo caché. En español se usan también, con este sentido, las expresiones antememoria o memoria intermedia.

Doc. 3 : données du CREA: I'exemple de cachet

Données statistiques

\begin{tabular}{|l|r|c|}
\hline \multicolumn{1}{|c|}{ Año } & \multicolumn{1}{c|}{$\%$} & Casos \\
\hline $\mathbf{2 0 0 1}$ & 14,28 & 4 \\
\hline 1991 & 10,71 & 3 \\
\hline 1992 & 10,71 & 3 \\
\hline 1997 & 10,71 & 3 \\
\hline 2002 & 10,71 & 3 \\
\hline 1994 & 7,14 & 2 \\
\hline 1995 & 7,14 & 2 \\
\hline 2000 & 7,14 & 2 \\
\hline 1977 & 3,57 & 1 \\
\hline Otros & 17,85 & 5 \\
\hline
\end{tabular}

\begin{tabular}{|l|c|c|}
\hline \multicolumn{1}{|c|}{ País } & \multicolumn{1}{c|}{$\%$} & Casos \\
\hline España & 46,87 & 15 \\
\hline Argentina & 40,62 & 13 \\
\hline Colombia & 3,12 & 1 \\
\hline México & 3,12 & 1 \\
\hline Perú & 3,12 & 1 \\
\hline Venezuela & 3,12 & 1 \\
\hline
\end{tabular}

\begin{tabular}{|l|c|c|}
\hline \multicolumn{1}{|c|}{ Tema } & $\%$ & Casos \\
\hline 4.- Artes. & 46,87 & 15 \\
\hline 2.- Ciencias sociales, creencias y pensamiento. & 21,87 & 7 \\
\hline 9.- Oral. & 12,50 & 4 \\
\hline 5.- Ocio, vida cotidiana. & 9,37 & 3 \\
\hline 7.- Ficción. & 6,25 & 2 \\
\hline 3.- Política, economía, comercio y finanzas. & 3,12 & 1 \\
\hline
\end{tabular}

\section{Occurrences en contexte (32)}

1 tan poco, que yo propuse al productor aportar mi 'cachet' al filme y llevarme un porcentaje de los posi ${ }^{* *} 1994$ PRENSA La Vanguardia, 10/03/1994: «Nunca tuve ambición artística». ESPAÑA 02.Astrología y ciencias ocult T.I.S.A (Barcelona), 1994

2 oma recaudó la suma necesaria para el pago de su «cachet». - Redacción. ${ }^{*} 1994$ PRENSA La Vanguardia, 27/02/1994: La Fira del Disc de Girona avanz ESPAÑA 04.Música T.I.S.A. (Barcelona), 1994

3 portables actrices. Con sus sesenta películas, el cachet y el gancho de Victoria Abril ponen los pelos ${ }^{\star *} 1990$ PRENSA Tiempo, 22/01/1990: APOGEO ESPAÑA 04.Cine y vídeo Ediciones Tiempo (Madrid), 1990

4 or viajeros ilustres, quienes disertaban sobre el cachet del viejo mundo, o los vestigios de imperios $v^{* *} 1996$ PRENSA El Tiempo, 06/09/1996: El cuento del sapo COLOMBIA 02.Ética (Bogotá), 1996

5 y fiestas electorales lo hacen contratados por un cachet normal. En los actos apartidistas algunos cant ${ }^{* *} 1986$ PRENSA El País, 01/10/1986: CANCION ESPAÑA 04.Música Diario El País, S.A. (Madrid), 1986

6 riores y su excelente educación, le han valido un cachet nada despreciable. DESMANES.- La ayuda económi ${ }^{* \star} 1995$ PRENSA El Mundo, 03/12/1995: Tanzania. Elecciones presidenciales. ESPAÑA 03.Política Unidad Editorial (Madrid), 1995 
7 emos tenido. Pero hay que tener en cuenta que los cachet de los grupos, sin ser muy altos -de unas 100. ${ }^{* *} 2001$ PRENSA La Ratonera. Revista asturiana de Teatro, mayo 2001: Admini ESPAÑA 04.Teatro Oris Teatro (El Entrego), 2001

8 rupo con el espectador? Esto se produce porque el cachet está asegurado y no depende de que el cliente ${ }^{* *} 2001$ PRENSA La Ratonera. Revista asturiana de Teatro, mayo 2001 : Entrev ESPAÑA 04.Teatro Oris Teatro (El Entrego), 2001

9 hasta ahora, a contratar actividades de muy bajo cachet, la mayor parte amateur; el Jovellanos no se $d{ }^{* *} 2000$ PRENSA La Ratonera. Revista asturiana de Teatro, 11/2000: La izqui ESPAÑA 04.Arte y cultura en general Oris Teatro (El Entrego), 2000

10 nfiesa el humorista-, sobre todo para arreglar el cachet. Además, Varela tiene -de su primer matrimonio- ${ }^{\star \star} 2000$ PRENSA Clarín, 12/01/2000: Parejero ARGENTINA 04.Teatro (Buenos Aires), 2000

11 bajal», ausente en esta edición por cuestiones de cachet. Los versos de Somos nosotros, su declaración ** 2002 PRENSA Clarín, 22/01/2002: HORACIO GUARANY FUE OVACIONADO ARGENTINA 04.Música (Buenos Aires), 2002

12 nterior conducción cobró por adelantado parte del cachet y eso los complicó. Les explicamos que no tene ${ }^{* *} 2002$ PRENSA Clarín, 22/01/2002: RIVER: AGUILAR NEGOCIA CON EL PLANTEL Y ARGENTINA 05.Deportes (Buenos Aires), 2002

13 sa de Ballas. Porque ellos solo se encargarán del cachet de Rojas. Como ayer había dudas sobre el arreg ** 1987 PRENSA Clarín, 21/10/1987: ARGENTINA 05.Deportes (Buenos Aires), 1987

14 caudado con River en Avellaneda. Y además está el cachet televisivo, cuyas cifras aún no se conocen, (e ${ }^{* *} 1997$ PRENSA Clarín, 09/05/1997: COPA LIBERTADORES: EN AVELLANEDA HACEN ARGENTINA 05.Deportes (Buenos Aires), 1997

15 naron los 50.000 dólares que Clarín les pagó como cachet al Hospital de Niños Ricardo Gutiérrez. Paso a ${ }^{\star *} 1997$ PRENSA Clarín, 14/02/1997: QUINTA EDICIÓN DEL GRAN DT ARGENTINA 04.Publicidad (Buenos Aires), 1997

16 ala y también Perú. El Che S.A. EL Che tiene más 'cachet' que nunca. Es 'chic' y se ha convertido en un ${ }^{* \star} 1997$ PRENSA Caretas, 09/10/1997: Yo Enterré AL CHE PERÚ 02.Historia (Perú), 1997

17 e ha dicho que se lo diga yo y que le pregunte el cachet. No sé muy bien cuánto cobra, cuánto ¿le dais ** --- ORAL Lo más plus, 13/12/96, Canal Plus ESPAÑA 09.Magacines ---

18 e podía ofrecer dos millones de libras que era su cachet, lo aceptó y fue realmente una un momento para ${ }^{*}$--- ORAL Lo más plus, 11/12/96, Canal Plus ESPAÑA 09.Magacines --19 e todos piden que les preste su belleza. Tiene el cachet más alto del mundo por sesión de fotos y a pes ** --- ORAL Hoy es posible, 28/01/96, TVE 1 ESPAÑA 09.Magacines ---

20 lmente importante, bonita, residencial y de mucho cachet en la ciudad. Este, nosotros teníamos familia ${ }^{* *}$--- ORAL CSHC-87 Entrevista 61 VENEZUELA 09.FORMALIDAD=baja, AUDIENCIA $=$---

21 co de Strawinsky salía con la suya, yo pediría un cachet. Cuatro mil pesos (de entonces) por mi actuaci ${ }^{\star \star} 1977$ Ocampo, Victoria Testimonios. Décima Serie. 1975-1977 ARGENTINA 02.Testimonios varios Sur (Argentina), 1977

22 andó a su representante Rodrigo Danús a exigir un cachet más alto, que quedó fijado en 35 mil dólares y ${ }^{* *} 2001$ Wornat, Olga Menem-Bolocco, S.A. ARGENTINA 02.Testimonios varios Ediciones B Argentina (Buenos Aires), 2001

23 cerca de 800.000 dólares entre sueldo del canal, cachet por presentaciones en festivales y los reporta ${ }^{* *} 2001$ Wornat, Olga Menem-Bolocco, S.A. ARGENTINA 02.Testimonios varios Ediciones B Argentina (Buenos Aires), 2001 
24 enir de la capital de jalisco diese un particular cachet a la profesión más vieja del mundo. No se deja ${ }^{* *} 2002$ Fuentes, Carlos En esto creo MÉXICO 02.Testimonios varios Seix Barral (Barcelona), 2002

25 s por su intervención. Ése era entonces el máximo cachet que podíamos pagar. Siete meses después ya dis ** 1992 Rodríguez Márquez, Nacho; Martínez Uceda La televisión: historia y desarrollo ESPAÑA 04.Medios de comunicación Mitre/Rtve (Barcelona), 1992

26 figuras de la televisión y disfrutan de un mayor cachet económico. Luis del Olmo, José María García, I ** 1992 Díaz, Lorenzo La radio en España (1923-1993) ESPAÑA 04.Medios de comunicación Alianza Editorial, S. A. (Madrid), 1993

27 rimorosa. Lo eran casi todo en la radio. Le daban cachet a la empresa, eran sus más señalados referente ${ }^{*} 1992$ Díaz, Lorenzo La radio en España (1923-1993) ESPAÑA 04.Medios de comunicación Alianza Editorial, S. A. (Madrid), 1993

28 o más serio, más destinado a poder cobrar un buen cachet y con más visión de futuro: empezó a tocar reg ** 1991 Polimeni, Carlos Luca ARGENTINA 04.Música AC (Buenos Aires), 1999

29 a seis conciertos y doscientos cincuenta pesos de cachet: por primera vez las Babys iban a cobrar. El $2^{* *} 1991$ Ramos, Laura; Lejbowicz, Cynthia Corazones en llamas. Historias del rock argentino en los '80 ARGENTINA 04.Música Clarín Aguilar U.T.E. (Buenos Aires), 1992

30 ofrecen posibilidades de aumentar sus ventas o su cachet. Pero una nueva concepción del arte global lat ${ }^{* *} 1991$ Fernández Chiti, Jorge Estética de la nueva imagen cerámica y escultórica ARGENTINA 04.Artesanía Condorhuasi (Buenos Aires), 1991

31 Evita como heroína histórica. Se le paga el mayor cachet abonado hasta entonces por la radiofonía argen ${ }^{* *} 1995$ Posse, Abel La pasión según Eva ARGENTINA 07.Novela Planeta (Barcelona), 1995

32 rque me precio de original. Tengo talento, tengo "cachet», recito a veces a Mallarmé. LUCIANO Y CREONTE ** 1989 Nieva, Francisco Corazón de arpía ESPAÑA 07.Teatro Junta de Castilla-La Mancha (Toledo), 1991 